\title{
Meta
}

Journal des traducteurs

Translators' Journal

ROSE, Marilyn Gaddis (dir.) (1990): Language \&

Communication, Volume 10, Number 1, Special Issue:

Language, Communication and Translation, Pergamon Press

\section{Denise Campillo}

Volume 36, numéro 4, décembre 1991

URI : https://id.erudit.org/iderudit/002193ar

DOI : https://doi.org/10.7202/002193ar

Aller au sommaire du numéro

Éditeur(s)

Les Presses de l'Université de Montréal

ISSN

0026-0452 (imprimé)

1492-1421 (numérique)

Découvrir la revue

Citer ce compte rendu

Campillo, D. (1991). Compte rendu de [ROSE, Marilyn Gaddis (dir.) (1990):

Language \& Communication, Volume 10, Number 1, Special Issue: Language,

Communication and Translation, Pergamon Press]. Meta, 36(4), 671-675.

https://doi.org/10.7202/002193ar d'utilisation que vous pouvez consulter en ligne.

https://apropos.erudit.org/fr/usagers/politique-dutilisation/ 
ROSE, Marilyn Gaddis (dir.) (1990): Language \& Communication, Volume 10, Number 1, Special Issue: Language, Communication and Translation, Pergamon Press.

La revue Language \& Communication, qui se veut interdisciplinaire, consacre un numéro spécial à la traduction. La direction de ce numéro a été confiée à Marilyn Gaddis Rose, directrice du Center for Research in Translation de Binghampton (SUNY). Dans son introduction, M.G. Rose pose les paramètres du débat: la traduction existe, elle est possible, elle est nécessaire puisqu'elle est au cœur de la communication. Les articles qui composent ce numéro spécial, venus d'horizons divers, proposent un éventail de points de vue sur le champ de la traduction.

H. STEPHEN STRAIGHT, «To immortalize without destroying». À partir de définitions du verbe translate, l'auteur, qui est anthropologue, brode diverses réflexions autour du thème de la traduction. Malgré le vague de certaines déclarations sur des notions comme meaning ou content, et sur la différence entre traduction «prosaïque» et traduction poétique, Straight a le mérite de situer en termes clairs la traduction dans le phénomène plus vaste de la paraphrase, champ fertile pour la réflexion.

KATHLEEN CALLOW, «Muddy hands need clear heads or a tale of two interfaces». L'aporie de l'impossibilité de traduire fait encore couler beaucoup d'encre, tout comme le débat sans fin sur les rapports entre traduction et linguistique. L'auteure cherche plutôt à répondre à la question: «Why can we not explain translation?» Elle situe le problème à deux interfaces: sens-langue d'une part, sémantique-pragmatique d'autre part. Le sens n'appartient pas aux mots, ni à une langue; il est «conceptual-experiential». Par la traduction, il franchit la barrière des langues, qui le structurent de manière différente; les traducteurs ont besoin que les linguistes se penchent sur cette question. La pragmatique, en replaçant les locuteurs dans la linguistique, rejoint les traducteurs qui savent qu'il n'y a pas de sens sans contexte. Callow réfute toutefois la prémisse selon laquelle la sémantique préexisterait à la pragmatique : on ne peut pas libérer tous les sens de leur contexte puis réintroduire le contexte à volonté. Cette carence fondamentale fait que l'interface sémantique-pragmatique ne présente pas pour la traduction l'intérêt théorique espéré. Retour donc à la sémantique, mais à une sémantique placée dans une perspective de communication.

WOLFRAM WILSS, «Cognitive aspects of the translation process». La traductologie doit établir un système de représentation qui objectivise les réflexions sur la problématique de la traduction. La psychologie cognitive lui offre un «concept central» placé dans une perspective de recherche interdisciplinaire et unifiée. La traduction est un type 
particulier d'activité linguistique combinant une série d'opérations mentales. Pour décrire et expliquer ces opérations, il faut faire appel à des concepts tels que l'action, le comportement, la résolution de problèmes, la prise de décision, la créativité, l'intuition, et examiner les stratégies mises en ouvre pour effectuer la traduction. Ce texte touffu offre une suite de réflexions théoriques et pratiques sur certains aspects pertinents de la psychologie cognitive et de l'analyse de discours; il signale l'impossibilité d'établir un modèle qui rende compte dans tous les cas de l'opération de traduction; il souligne enfin que la traductologie n'a pas besoin d'un «masque conceptuel» mais d'une théorie de la connaissance pratique.

EUGENE A. NIDA, «The role of rhetoric in verbal communication». Cet article n'est pas axé sur la traduction, mais nous y ramènera. Le célèbre Nida reprend ici en les enrichissant les fonctions du langage (expressive, cognitive, informative, impérative, performative, émotive, interpersonnelle), et rappelle qu'un discours combine généralement plusieurs de ces fonctions. D'autre part, les processus fondamentaux de l'usage d'un code, sélection et arrangement, sont à voir à deux niveaux: «macrolevel» (macrostructure?), thèmes et acteurs, type de discours, niveau de langue, et «microlevel» (microstructure?), éventail de possibilités thématiques et de procédés rhétoriques. Ces principes de sélection et d'arrangement, au niveau du fond et de la forme, doivent établir un équilibre entre impact et attrait:

_-impact: nouveauté, pertinence, réalisme;

— attrait : - fond - complétude, proportion, cohérence ;

- forme - adéquation, cohésion, relief;

- clarté.

Les procédés rhétoriques, tant formels que sémantiques, permettent d'enrichir l'impact et l'attrait du discours. Quel est leur intérêt pour la traduction? Les traducteurs doivent être conscients des diverses fonctions, au sein d'une langue et surtout dans le passage d'une langue à l'autre, que peuvent assumer les procédés rhétoriques.

R.R.K. HARTMANN, «The not so harmless drudgery of finding translation equivalents». Un lexicographe se penche sur la notion d'équivalence dans la perspective de la lexicographie bilingue. Qu'est-ce que l'équivalence? Les traductologues n'ont pas su jusqu'ici formuler des principes clairs justifiant le choix des équivalents. L'auteur rappelle les faiblesses des analyses structuralistes, et souligne la nécessité de faire appel à la sociolinguistique et à la psycholinguistique et de placer la lexicographie dans un cadre sémiotique qui tienne compte des normes linguistiques et culturelles du lecteur.

Est-il possible d'établir une classification des types d'équivalence sans référence aux stratégies mises en cuvre par les locuteurs bilingues? Observant la démarche des lexicographes, l'auteur conclut que traduction, linguistique comparée et lexicographie bilingue partagent le même champ paradigmatique. Comment le compilateur d'un dictionnaire bilingue fixe-t-il les équivalents lexicaux? La réponse pourrait être dans le dépouillement de textes parallèles qui fourniraient des équivalents dans leur contexte naturel. Ces équivalents en contexte le resteraient-ils hors contexte? Les relations d'équivalence sont anisomorphiques et asymétriques. Il est donc très difficile de prédire l'usage des mots en contexte et pratiquement impossible de présenter des équivalents de manière parfaitement bidirectionnelle dans un dictionnaire bilingue. Quelles opérations sont à la disposition du lexicographe pour trouver les équivalences? Comment les étudier et en enseigner l'emploi ? L'auteur relève les diverses opérations en jeu dans la préparation des dictionnaires bilingues: substitution, transposition, modulation, transfert, emprunt, adaptation, circonlocution. La paraphrase (expression d'un même sens sous des formes différentes dans une même langue) et la métaphrase (changement de code linguistique) 
sont des moyens fondamentaux auxquels a recours le traducteur et qui doivent figurer parmi les priorités de la recherche. Lexicographie et traduction doivent se féconder l'une l'autre, notamment par l'intermédiaire de l'ordinateur.

JO ANNE ENGELBERT, «Neither Hades nor Hell: problems of allusion in the translation of Central American poetry». Qui connaît le Popol-Vuh, le livre sacré des Mayas? Devant une allusion à ce texte dans le poème d'un auteur d'Amérique Centrale, quelle stratégie doit adopter le traducteur? Dans un souci de transparence et d'universalité, va-t-il choisir une référence à la culture européenne? Engelbert nous montre que les enjeux se trouvent en fait à un autre niveau. L'allusion est tout aussi obscure pour la plupart des lecteurs hispanophones que pour ceux des autres cultures. Le poète, en faisant revivre les éléments d'une ancienne cosmogonie, accomplit un acte de libération historique contre la colonisation européenne qui a presque effacé toute référence à la culture de ses ancêtres. En même temps qu'elle joue ce rôle d'éveil à l'histoire ancienne, l'allusion renvoie en outre à l'histoire récente; l'extermination par les colonisateurs européens devient métaphore de l'oppression qui règne à notre époque dans certains pays; l'évocation des souffrances anciennes devient donc un outil de résistance politique.

Pour le traducteur, le travail de compréhension est double, puisqu'il lui faut connaître aussi bien le passé que le présent pour saisir l'allusion. Mais jusqu'où doit aller l'exégèse? Faut-il lever les ambiguittés? Dans la traduction de la poésie engagée, Engelbert propose de respecter au maximum les allusions, même les plus étrangères au savoir ou à la culture du lecteur, mais de mettre à la disposition de ce dernier un arsenal d'outils de décodage qui sont les instruments classiques de l'exégèse: préface, gloses, glossaire, chronologie, notes (discrètes). Cette stratégie permet d'établir un pont entre les cultures, et donc peut-être de cheminer sur la voie d'une véritable universalité.

RIA VANDERAUWERA, «What translation can (and should) do for little-known languages». Quel rayonnement peut avoir la littérature d'une langue dont le bassin est réduit, comme le flamand, qui est parlé aux Pays-Bas, en Flandre, dans les Antilles néerlandaises et au Surinam? La diffusion des ouvrages anglo-américains dans ces pays est bien établie, mais peut-on aller à contre-courant et faire connaître des cuvres flamandes aux États-Unis, par exemple? C'est la vocation que s'est donnée la Fondation pour la traduction des œuvres littéraires flamandes, financée par les Pays-Bas et la Belgique. La Fondation aide les auteurs à trouver un éditeur étranger, en payant la première traduction (parfois sommaire, et simplement destinée à faire juger de la qualité de l'œuvre) dans une langue de large diffusion, et en offrant d'acheter un certain nombre d'exemplaires du livre. La traduction à partir de langues à faible diffusion pose des problèmes: il est difficile de trouver des traducteurs maîtrisant parfaitement deux langues et deux cultures; la solution est souvent dans la collaboration. La Fondation apporte son aide sous forme de rémunération, d'organisation d'ateliers, de financement de voyages, d'aide à la documentation. Restent la promotion et la vente; il est difficile de vendre un livre venant d'un pays qui ne fait pas les manchettes, à moins de travailler dans un genre bien délimité ( «réalisme magique» d'Amérique latine, roman noir ou policier, etc.). Bien des auteurs et chercheurs flamands écrivent directement en anglais. Un espoir toutefois: la renaissance des petites maisons d'édition et la vigueur des presses universitaires ouvrent un nouveau champ d'expression aux littératures minoritaires. L'effort de promotion mené aux États-Unis par la Fondation met en lumière des auteurs flamands, et l'opération a des retombées plus larges grâce à la traduction de leurs œuvres vers d'autres langues.

ANDRÉ LEFEVERE, «Ibi sint traductores: translation as communication in and about Africa». L'Afrique compte 800 à 1000 langues différentes, qui ne ressemblent en rien aux langues européennes de la colonisation. Pour le traducteur, sans même parler du 
ton, caractéristique intraduisible de la plupart de ces langues, il est des éléments avec lesquels il faut composer. La syntaxe, fort éloignée des syntaxes européennes, exige un choix: adaptation ou pidginisation? Les références culturelles à des objets ou des concepts peuvent être déroutantes; le traducteur devrait pouvoir donner au lecteur, par une introduction ou une postface, les explications lui permettant de relativiser les cultures.

Les littératures africaines risquent de mourir. Euvres d'oralité, qui souvent refusaient d'être figées dans l'écrit, elles ont été niées dans leur valeur et leur spécificité. La volonté de les préserver n'est apparue que récemment parmi les élites africaines. Il est urgent de recueillir, de transcrire, de préserver, de publier et de traduire la littérature orale «classique», du moins tant qu'il reste des conteurs pour la dire, et même si sa fixation dans l'écrit ou son passage dans des langues étrangères peuvent être considérés comme une trahison. En regard apparaît une nouvelle littérature, écrite par des Africains dans des langues non africaines. Ce produit de l'élite africaine ne sera reçu que par des lecteurs maîtrisant la culture euro-américaine, à moins d'être traduit dans une langue africaine. Lefevere dessine deux voies parallèles: la littérature classique doit être traduite en langues non africaines, tandis que la nouvelle littérature africaine d'expression euroaméricaine doit être traduite dans les langues africaines. Les deux voies exigent courage et honnêteté intellectuelle.

CHARLES A. NAMA, «A critical analysis of the translation of African literature». Dans cet article composé, semble-t-il, de passages tirés d'un livre encore inédit sur l'histoire de la traduction et de l'interprétation en Afrique (ce qui lui donne un caractère décousu), l'auteur situe son propos, la nécessité de théoriser la traduction littéraire africaine, dans un cadre historique. Au XVe siècle, les colonisateurs apportent l'écriture, ce qui favorise l'émergence d'une littérature tant en langues européennes qu'en langues africaines, et un essor de la traduction et de l'interprétation. On note au XIX'e siècle une importante production en portugais, notamment en Angola, où paraît un journal bilingue portugais-kimbudu. De grands traducteurs-terminologues ont su, pendant l'époque coloniale, établir des dictionnaires bilingues qui ont stimulé le travail linguistique sur le continent. Après l'indépendance, on note le développement du kiswahili comme langue véhiculaire, et donc comme langue de traduction en Afrique orientale. Dans l'espace linguistique africain, les approches théoriques de la traductologie euro-américaine sontelles pertinentes? Nama souligne les carences des démarches strictement linguistiques et la nécessaire fonction herméneutique de la traduction. Certains poètes africains, écrivant à la fois dans une langue africaine et dans une langue européenne, présentent les deux versions en regard, assorties d'explications et d'observations. Cette approche socioculturelle ouvre l'œuvre aux lecteurs, quels qu'ils soient, et appelle les commentaires de ceux qui maîtrisent les deux langues. On observe par contre, selon Nama, des traductions, faites par des Euro-Américains, qui sont truffées d'erreurs et de faiblesses, faute d'une connaissance suffisante des réalités linguistiques, culturelles, sociales ou historiques chez les traducteurs. Les exemples donnés ici par Nama sont malheureusement loin d'être convaincants: il s'agit d'une traduction de l'anglais vers le français, et d'une du français vers l'anglais; leurs faiblesses semblent être d'ordre purement lexical. C'est certainement en amont, dans la traduction d'une langue africaine vers une langue européenne, ou dans le choix que fait un écrivain africain de s'exprimer directement dans une langue européenne, que doit entrer en ligne de compte la dimension socioculturelle, si importante dans le contact entre une littérature africaine et une culture européenne.

La «théorisation» de la traduction littéraire africaine, que l'auteur de l'article appelle de tous ses vœux, devrait peut-être avant tout: 
- être une expansion des recherches traductologiques actuelles, qui concernent essentiellement les langues européennes;

- intéresser d'autres langues et cultures non européennes;

- fixer les paramètres de son champ d'étude, en différenciant par exemple la littérature d'expression africaine de la littérature africaine d'expression européenne.

HANS-GEORG GADAMER, «Hearing - seeing — reading». C'est ici le philosophe qui parle. La lecture est proche de l'écriture, et donc du langage. La lecture est impossible sans compréhension; de même, la parole doit être entendue. La vue et l'ouïe sont des sens souvent rivaux mais complémentaires, et qui s'unissent dans la lecture: il faut passer par l'ouïe (le langage) pour comprendre ce que la vue perçoit. Ce n'est que depuis peu, dans la culture européenne, qu'on lit sans accompagnement oral. On note un aller-retour dans la tradition épique entre l'écrit et l'oral qui s'imposent mutuellement leurs contraintes. La lecture, orale ou silencieuse, éveille des images, une «perception intuitive», une représentation sur la «scène intérieure» de Goethe. Gadamer nous renvoie à diverses conceptions de la poésie, des métaphysiciens grecs à Derrida en passant par Kant et Heidegger, conceptions qui tournent autour du rapport au temps, de la perception du temps. La lecture fait appel à la fois à la simultanéité (perception intuitive de la «présence», anticipation du sens, unité) et à la succession (manifestation physique du temps, suite des mots). Dans le langage, certains éléments stabilisateurs nous donnent prise sur l'unité du texte, unité présente dans le mot, unité créée par la vuc et l'ouïe qui, ensemble, participent à la lecture, et permettent au lecteur d' «habiter» l'œuvre.

Ces remarques d'ordre philosophique, fort éloignées du champ de réflexion habituel des traducteurs, ont peu de pertinence directe en traductologie; elles peuvent cependant être instructives, et cela à deux titres. D'une part, elles nous rappellent que la philosophie s'est toujours intéressée au langage, à la communication, à la compréhension, à l'intuition - qui sont au cœur de l'opération traduisante. D'autre part, elles nous font voir combien il est difficile d'appréhender le phénomène de la lecture, ce rapport à l'écrit dans lequel le traducteur est en position particulière, puisqu'il est le seul lecteur à devoir rendre compte de manière exhaustive de sa perception.

DeNISE CAMPILLO 